Beverley M. Clarke

Adrian R.M. Upton

Mark V. Kamath

Talal Al-Harbi

Claudia M. Castellanos

\title{
Transcranial magnetic stimulation for migraine: clinical effects
}

Received: 25 April 2006

Accepted in revised form: 26 June 2006

Published online: 25 October 2006

B.M. Clarke • A.R.M. Upton • M.V. Kamath

T. Al-Harbi • C.M. Castellanos

Division of Neurology,

McMaster University,

Hamilton, Ontario, Canada

B.M. Clarke ( $\square)$

Hamilton Health Sciences Centre,

Room 4U6,

Department of Clinical Neurology,

McMaster University,

1200 Main Street West,

Hamilton, Ontario, Canada L8N $3 Z 5$

e-mail: clarke3@mcmaster.ca

Tel.: +1-905-521-2100 ext.76368

Fax: +1-905-521-2656

\author{
Abstract The objective was to \\ assess the impact of transcranial \\ magnetic stimulation (TMS) on \\ pain and the autonomic nervous \\ system (ANS) in migraine. Forty- \\ two people [mean age $41.43 \pm 11.69$ \\ (SD) years, 36 females] were ran- \\ domised into high $v s$. low TMS \\ stimulation groups and received 2 \\ brief pulses of TMS. Thirty-three \\ (33/42) individuals had heart-rate \\ variability assessed, before and \\ after stimulation. No group effects \\ were found. Pain decreased by $75 \%$; \\ $32 \%$ of people after 1 treatment \\ reported no headache after $24 \mathrm{~h}$. \\ Mean heart rate decreased from \\ $79.05 \pm 10.27$ to $72.89 \pm 11.35$
}

beats/min. The low-frequency (LF) and the high-frequency (HF) areas derived from power spectral analyses increased [mean $6522 \pm 1277$ to $8315 \pm 1009$ beats $/ \mathrm{min}^{2}$ (LF) $(p=0.001)$ and mean $5600 \pm 1568$ to $8755 \pm 3071$ beats $/ \mathrm{min}^{2}$ (HF) $(p=0.001)]$. The LF:HF ratio decreased from mean $1.31 \pm 0.51$ to $1.13 \pm 0.48$ (NS). TMS produces immediate, sustained reductions in pain and modification of the ANS.

Keywords Migraine $\cdot$ Transcranial magnetic stimulation $\cdot$ Pain

\section{Introduction}

Burden of illness and patient satisfaction reports in migraine headache show that current treatment regimes are incomplete $[1,2]$. The main patient concerns are that (a) relief of pain is not immediate or sustained, (b) potential drug side effects are worrisome, and (c) the frequency of headache recurrence is not abolished or significantly diminished. As at least $10 \%$ of the population worldwide suffers from migraine headache, the impact of these problems is significant [3]. More effective treatment regimes and a complete understanding of the pathophysiology of migraine are needed.

Controversial theories about the cause and mechanisms of migraine range from experiments that show impaired brain energy metabolism [4], autonomic nervous system (ANS) hypofunction [5, 6], brain cell hyperexcitability to low intracellular brain magnesium [7-15]. Once considered solely a vascular event, current research supports a combination of both neural and vascular causes [16-20]. Many studies report the biochemical reaction and clinical effects of medications in migraine [21].

Despite considerable study, a unified theory of the pathophysiology of migraine does not exist. The most current cortical depression theories argue that the spreading depression of Leão is related to glutamate changes within the brain. This mechanism may not apply to all forms of migraine, and studies of cortical excitability of migraine done between migraine attacks yield conflicting results concerning the hypo- and hyperexcitability of the cortex [22-33]. Because 
both pharmacological and non-pharmacological treatment interventions only partly address patient concerns, a novel treatment method using single-pulse transcranial magnetic stimulation (TMS) was evaluated.

\section{Justification for the application of TMS}

TMS involves the application of a large brief current that is passed through an insulated coil placed on the scalp. The current sets up a magnetic field that penetrates the skull producing a secondary current that stimulates the cerebral cortex as well as trigeminal nerve fibres. This secondary current alters the cortical excitability.

Theoretical mechanisms of the relationship between migraine headache with aura and TMS are that just before the headache begins, brain cells become overly active and form a wave of increased neuronal activity. The travelling wave of hyperactive cells spreads across the cortex followed by an area of depressed neuronal activity. The effect of this process may be the occurrence of an aura, pain, sensory changes in the face and/or limbs, and/or nausea and dizziness, through activation of the trigeminal-vascular system and the trigeminal-cervical complex [34]. It is hypothesised that if very short pulses (1 ms) of TMS are applied to the scalp just as the headache begins, these pulses stop the travelling wave of hyperactive brain cells and subsequent neuronal depression. The headache either stops or it is less severe. Repeated applications may result in a decrease in headache frequency or complete cessation of headache.

Safety data indicate that single-pulse transcranial stimulation is a safe, useful tool for investigating neurophysiological mechanisms in human studies. No adverse side effects with single-pulse stimulation have been reported $[35,36]$.

We report the results of a study to assess the efficacy of TMS on pain intensity and heart-rate variability (HRV) in individuals with migraine headache.

This project was approved by the Research Ethics Committee of the Hamilton Health Sciences Centre, Hamilton, Ontario, Canada. An information package was provided and informed consent was obtained from all participants, and the study adhered to the principles established in Helsinki.

\section{Patients and methods}

\section{Sample}

Eighty (80) people from the community-at-large were screened for admission to the study. All individuals had been previously diagnosed with migraine and were re-assessed at intake.
Forty-two (42) individuals with migraine (ICHD-II classification) met the inclusion criteria [37]. One of the criterion was that individuals must live and/or work within $30 \mathrm{~min}$ of our laboratory. This factor was important because it was necessary to apply the stimulation as soon as possible. Exclusion criteria were metal in the cranium, cardiac/neuro pacemakers, previous seizure activity, neurosurgery or head injury. Ten (10) people had migraine with aura, 25 without aura and 6 had headache with migraine components. The average headache duration was $8.84 \pm 14.65(\mathrm{SD}) \mathrm{h}$. Headache pain persisted, on average, from $20 \mathrm{~min}$ to 10 days. The shortest episode was 10 $\min$ and the longest 31 days. Headache frequency ranged from $1 /$ day to $4 /$ year. In 25 patients precipitating factors were unknown. In the remaining patients $(n=17)$, hormonal changes $(n=6)$, stress $(n=2)$, head injury $(n=3)$ and food sensitivities $(n=2)$ were thought to be precipitating factors for migraine. Fourteen people reported onset of headache in the morning, 11 people reported the afternoon as time of occurrence and 17 were unable to define a pattern of onset. Patients (mean age of $41.42 \pm 11.69$ years, 36/42 females) were randomised into either the high stimulation (50\% of the maximum output) or low stimulation (30\% maximum output) group. Of those individuals who received stimulation, five people had an aura and three of the five had pain with an aura. There were 19 complaints of pain in the temporal region, 24 in the occipital area and 8 in the frontal area of the brain. Nine out of 51 complaints were in more than one area. Other signs and symptoms were nausea, double vision, photophobia, aura and dizziness. Headache diaries were kept pre- and post-stimulation. There were no restrictions on medications, which consisted of analgesics, narcotics, antiemetics and/or sedatives.

\section{Measurements}

\section{Stimulation parameters}

TMS was applied using a Caldwell Stimulator, model \#MES-10. The stimulator was programmed to deliver a pulse of $70 \mu \mathrm{sec}$ width. The coil radius was $9.5 \mathrm{~cm}$. Peak induced voltage was $187 \mathrm{~V}$. The magnetic intensity in the middle of the coil was $2.3 \mathrm{~T}$. TMS was applied as soon as possible on arrival in the laboratory. Each person received two brief pulses, $5 \mathrm{~s}$ apart, over the area of perceived pain or over the area of the brain generating the aura. Patients and examiners were blinded as to the intensity of the stimulation (high $v s$. low). Forty-two people received 1 trial, 22/42 had 2 trials and 11/42 people had 3 trials. No person received more than 3 impulses of TMS at any given time. Repeat trials remained at the initial stimulation intensities. Follow-up calls were made to individuals $24 \mathrm{~h}$ after stimulation.

Pain and suffering measures

Pain intensity was measured using a 5-point Likert-type scale in which 5 was the worst response. Pain levels were recorded prestimulation, and at post-stimulation at 5-min intervals for 20 min. Suffering was assessed using the Measuring and Assessing Suffering Questionnaire (MASQ) on intake to identify the suffering component [38].

\section{Heart-rate variability measures}

Thirty-three (33/42) individuals were assessed for ANS effects using HRV measures. Nine people who were part of the pilot 
study were not assessed for HRV. HRV was measured 5 min before stimulation and for $20 \mathrm{~min}$ post-stimulation. The HRV signal was computed from the electrocardiogram (ECG) and subjected to spectral analyses. Areas under the low-frequency (LF) and high-frequency (HF) components of the HRV were determined and a ratio of the powers (LF:HF area) was calculated.

\section{Signal processing of heart rate variability}

Processing the HRV was done as follows. A continuous ECG signal was obtained from the lead II electrodes, amplified and sampled at 500 times/s and stored on a hard disk [39]. The stimulation did not generate any ectopic beats. Off-line analysis of the power spectra of the heart rate (HR) was performed. Following QRS detection, the HR signals of $128 \mathrm{~s}$ were interpolated using linear interpolation every $0.5 \mathrm{~s}$. The re-sampled HRV signal was passed through a 4thorder high-pass IIR filter with a cut-off frequency of $0.02 \mathrm{~Hz}$ to remove the very low frequency components. Subsequently, an 11thorder autoregressive model was fitted to the signal and power spectra were computed using the autoregressive modelling method. Following normalisation, percentage areas in the LF band and the $\mathrm{HF}$ band were computed. The LF band was from 0.02 to $0.15 \mathrm{~Hz}$ while the HF band extended from 0.15 to $0.4 \mathrm{~Hz}$. The LF band is predominantly sympathetic and the HF band is vagally modulated [40]. A ratio of the LF:HF ratio was computed before and after stimulation. All results are expressed as mean \pm SD.

\section{Statistical analyses}

Analyses of variance techniques were applied to determine whether there were differences between the high- and low-stimulation groups. A repeated measures of covariance design was used to assess the mean difference in a Likert scale pain "score". The 4 paired observations for each study participant high $v s$. low (minute 5, 10, 15, 20) could effectively use each subject as his/her own control. A further specification (that of a covariate which remained constant across trials) was used to adjust for preintervention pain. The repeated measures ANOVA also originally included a between-subjects grouping factor of stimulation group (high vs. low intensity). The mean HR and indices derived from the power spectrum of the HRV were subjected to Student's t-test to determine if there was a significant difference before and after stimulation. A minimum level of significance was set at $p<0.05$.

\section{Results}

High vs. low stimulation

The mean differences between the low-stimulation and high-stimulation groups were found to be slight, even after adjusting for baseline (pre-intervention) pain. The interaction term for stimulation group by time (minute 5 to minute 20 post-stimulation) was not statistically significant. An apparent (albeit non-significant) linear trend was still found for a main reduction in pain from minute 5 to minute 20 post-stimulation. Further, data exploration revealed few outliers in either stimulation group. Subsequently, the high- and low-stimulation groups were combined into one group for further comparisons.

\section{Pain and suffering}

There was an overall mean decrease in pain intensity of $75 \%$ which was calculated based on the change between the pre-stimulation mean of pain intensity, 3.30 \pm 0.74 , and the post-stimulation value, $2.49 \pm 1.01 \quad(p<0.05)$. At the intake assessment there were no significant differences in total suffering scores between those persons with pain $(n=19)$ and those without $(n=23)$. In the pain group, mean values for total suffering were $2.24 \pm 0.54$ vs. $2.36 \pm 0.573$ $\mathrm{SD}$ for the no-pain group.

\section{Autonomic nervous system effects}

ANS effects were significant. Mean HR decreased from $79.05 \pm 10.27$ to $72.89 \pm 11.35$ beats $/ \mathrm{min}$. The LF area increased from $6522 \pm 1277$ to $8315 \pm 1009$ beats $/ \mathrm{min}^{2}$ $(p<0.001)$, and the HF area increased from $5600 \pm 1568$ to $8755 \pm 3071$ beats $/ \mathrm{min}^{2} \quad(p<0.001)$. The LF:HF ratio decreased from $1.31 \pm 0.51$ to $1.13 \pm 0.48$ (NS). An example of the power spectrum before and immediately after TMS, in one subject, is shown in Figures 1 and 2.

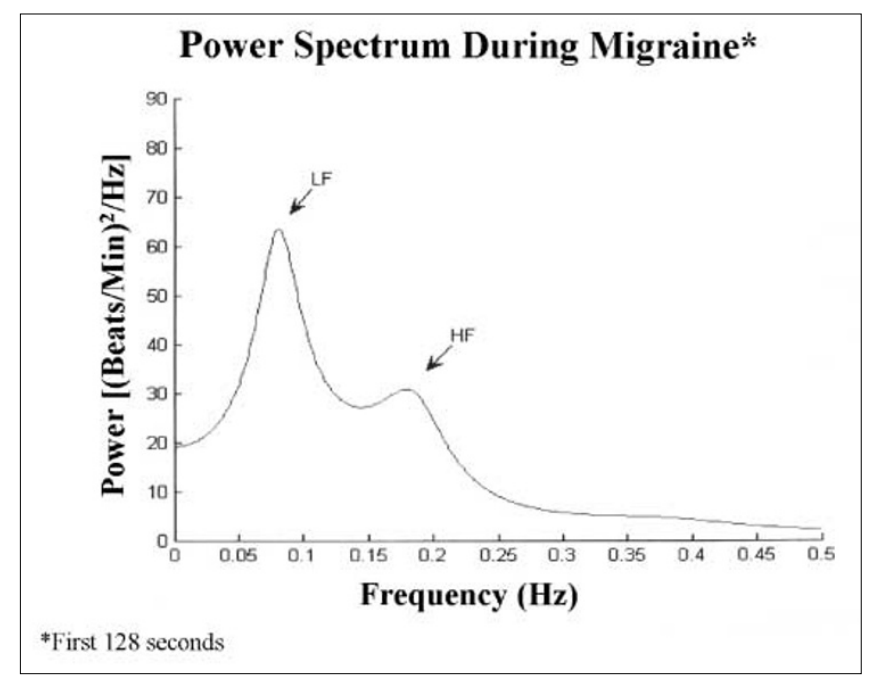

Fig. 1 A segment of the continuous heart-rate variability data of a patient during migraine. The length of the recorded HRV is approximately $300 \mathrm{~s}$ and the power spectrum of the first $128 \mathrm{~s}$ of the signal is plotted 


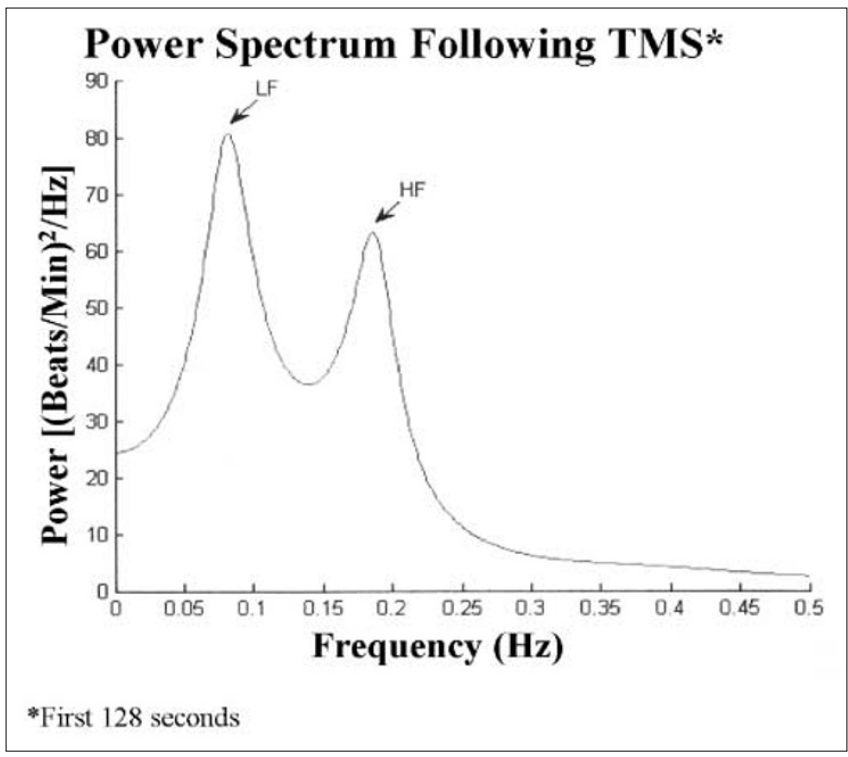

Fig. 2 Depicts the heart-rate variability signal of a patient following transcranial magnetic stimulation. The power spectrum of the first $128 \mathrm{~s}$ of the HRV signal is plotted. The mean heart rate shows a slight downward trend. However, note the increase in power in both LF and HF bands of the power spectrum

Table 1 TMS and headache recurrence after $24 \mathrm{~h}$

\begin{tabular}{lllrr}
\hline \multirow{2}{*}{ Trials } & \multicolumn{3}{c}{ Headache recurrence } \\
\cline { 2 - 5 } & None & Mild & Moderate & Severe \\
\hline $1(\mathrm{n}=42)$ & $32 \%$ & $24 \%$ & $11 \%$ & $33 \%$ \\
$2(\mathrm{n}=22)$ & $29 \%$ & $16 \%$ & $8 \%$ & $46 \%$ \\
$3(\mathrm{n}=11)$ & $40 \%$ & $30 \%$ & $0 \%$ & $30 \%$ \\
\hline
\end{tabular}

Results suggest that the effects of TMS may be cumulative

\section{Efficacy}

Sixty-nine percent of the people showed improvement with 1 trial, $87 \%$ improved in those persons who had 2 trials and $82 \%$ got better after 3 trials. In individuals with an aura, relief was $100 \%$ and immediate. The mean time to show improvement was $15.46 \pm 6.82 \mathrm{~min}$. Improvement was defined as a decrease in perception of pain of at least 1 level on a 5-point Likert-type scale, and as a decrease in the number of headaches occurring after TMS.

Of those persons who received 1 trial $(n=42), 32 \%$ reported no further headache after $24 \mathrm{~h}$. After 2 trials $(n=22), 29 \%$ were headache free the next day and in those persons $(\mathrm{n}=11)$ who had 3 trials, $40 \%$ reported no further headaches after $24 \mathrm{~h}$ (see Table 1). Of those persons without an aura who received 1 trial, 6 patients were headache free, of those who had 2 trials, 8 were headache free and after 3 trials, 1 was headache free after $20 \mathrm{~min}$.
Seventy-six percent of patients who received 1 trial did not take medications before stimulation. Twenty-four percent did not take medication in the following $24 \mathrm{~h}$. In the 2-trials group, 52\% did not take medication prior to stimulation and 35\% did not take rescue medication for $24 \mathrm{~h}$. For the group who received 3 trials, $55 \%$ did not take medication pre-stimulation and $60 \%$ did not take it for $24 \mathrm{~h}$.

Side effects

No adverse side effects were reported either immediately post-treatment or after $24 \mathrm{~h}$. Only 1 person reported slight unsustained dizziness and 1 person was a little drowsy. Several people reported feeling more energetic while others reported longer than usual times between headache onset. Two people said they felt tired after treatment. For those who did not receive immediate benefit with only 1 trial, reports were that there was difficulty controlling the persisting headache with their usual treatments. None of these effects were recurring or required medical attention.

\section{Discussion}

TMS is an important adjunct treatment for migraine headache. Pain relief is often immediate and frequency of headache recurrence decreased by $48 \%$ after 1 trial with no adverse side effects. Reports of improvement after more than 1 trial may be due to a cumulative effect of TMS. Further studies are needed.

Processing the HRV signals makes a significant contribution to the understanding of the role of the ANS in migraine. During the study we observed that TMS provided reduction in the pain. Our goal was to assess the effect of such changes objectively using PS/HRV. If one reduces the pain, there may be changes in the sympathetic-vagal balance of the ANS. Future studies are needed to compare the effects on the ANS between TMS and other interventions such as pharmacological agents.

Further, Appel et al. [40] report an enhancement of LF power and suggest that this finding is due to vagal nerve overflow to the sinus node, indicating that patients with migraine have sympathetic system instability. Our study shows that TMS produces perturbations of the ANS, which results in a balancing of the sympathetic and parasympathetic components. TMS may "normalise" the ANS and subsequently modify the vasodilatation of the meningeal blood vessels. These observations support the work of Silberstein [17], Goadsby [19] and Matharu et al. [13], who argue that balancing the ANS may not only prevent abnormal sensory neuron activation in the trigeminal 
nucleus caudalis, a process thought to be the cause of the phenomenon of cutaneous allodynia, but it may also have a damping effect on central nervous system hyperactivity and thereby abort the headache.

The relationship between TMS and headache medications also requires further investigation. Although $76 \%$ of individuals who received TMS did not take medication prior to treatment and $24 \%$ did not take medications posttreatment, the relationship between TMS and specific drug regimes must be rigorously delineated [41, 42].

One limitation of the study is that it was hypothesised that the low stimulation group would show no effect and as a result would act as a control group. As there is an effect in both groups, stimulation/sham stimulation studies are being done. Optimal stimulation intensities are now possible with the advent of newer stimulation devices. Patients and investigators are blind as to whether stimulation is being delivered or not. To explore the issue of placebo more rigorously, patients are initially asked whether they think they are in the stimulation or non-stimulation group and are questioned again after the intervention is applied. Results are pending.

A second limitation to the study is that patients were required to come to the laboratory and sometimes, by the time they arrived, the aura had disappeared and the headache was well established. A portable device has been developed to overcome this problem and it is currently being tested. We believe that further episodes of stimulation could have achieved better control of headache. Availability of the portable device will allow testing of this hypothesis.

Preliminary intake results also provide evidence that the phenomenon of suffering, in association with migraine headache, is separate from pain. These results confirm findings in other chronic illnesses (arthritis) [38]. Suffering is an issue to be addressed if optimal care in migraine is to be achieved. Perceptions of suffering were the same for those who had pain as those who did not. Follow-up measures of suffering, post-stimulation, would be useful and are incorporated into our new protocol.

To date, our initial results provide evidence that TMS can prevent headache and is an effective adjunct treatment for migraine headache pain. TMS meets the criteria of immediate, sustained pain relief with no known side effects. TMS produces a balancing of the sympathetic and parasympathetic components of the ANS.

Acknowledgements We acknowledge the financial support of the DeGroote Foundation and the National Sciences and Engineering Research Council of Canada (NSERC), and the assistance of Mary Lou Schmuck, Statistician, Program for Educational Research and Development, Faculty of Health Sciences, McMaster University, Hamilton, Ontario, Canada.

\section{References}

1. Gallagher RM (2004) What do patients want from acute migraine treatment? Cephalalgia 24[Suppl 4]:8-15

2. Massiou H (2003) Migraine medication attributes are important for patient compliance. Drugs Today 39[Suppl D]:25-29

3. Leonardi M, Steiner TJ, Scher AT, Lipton RB (2005) The global burden of migraine: measuring, disability in headache disorders with WHO's classification of functioning, disability and health (ICF). J Headache Pain 6:429-440

4. Montagna P, Cortelli P, Barbiroli B (1994) Magnetic-resonance spectroscopy studies in migraine. Cephalalgia 14:184-193

5. Schoenen J, Thomsen LL (2000) Neurophysiology and autonomic dysfunction in migraine. In: Olesen J, Tfelt-Hansen P, Welch KMA (eds) The headache. Lippincott, Williams and Wilkins, Philadelphia, PA
6. Shechter A, Stewart WF, Silberstein SD, Lipton RB (2002) Migraine and autonomic nervous system function - a population-based, case-control study. Neurology 58:422-427

7. Welch KMA, Barkley GL, Tepley N, Ramadan NM (1993) Central neurogenic mechanisms of migraine. Neurology 43[Suppl 3]:S21-S25

8. Fumal A, Bohotin V, Vandenheede M, Schoenen J (2003) Transcranial magnetic stimulation in migraine: a review of facts and controversies. Acta Neurol Belg 103:144-154

9. Aurora SK, Welch KMA (1998) Brain excitability in migraine: evidence from transcranial magnetic stimulation studies. Curr Opin Neurol 11:205-209

10. Aurora SK, Ahmad BK, Welch KMA et al (1998) Transcranial magnetic stimulation confirms hyperexcitability of occipital cortex in migraine. Neurology 50:1111-1114
11. Aguggia M, Zibetti M, Febbraro A, Mutani R (1999) Transcranial magnetic stimulation in migraine with aura: further evidence of occipital cortex hyperexcitability. Cephalalgia 19:465

12. Afra J, Mascia A, Gerard P et al (1998) Interictal cortical excitability in migraine: a study using transcranial magnetic stimulation of motor and visual cortices. Ann Neurol 44:209-215

13. Matharu MS, Bartsch T, Ward N et al (2004) Central neuromodulation in chronic migraine patients with suboccipital stimulators: a PET study. Brain 127:220-230

14. Avnon Y, Nitzan M, Sprecher E et al (2003) Different patterns of parasympathetic activation in uni- and bilateral migraineurs. Brain 126:1660-1670

15. Avnon Y, Nitzan M, Sprecher E et al (2004) Autonomic asymmetry in migraine: augmented parasympathetic activation in left unilateral migraineurs. Brain 127:2099-2108 
16. Thomsen LL, Iversen HK, Boesen F, Olesen J (1995) Transcranial doppler and cardiovascular-responses during cardiovascular autonomic tests in migraineurs during and outside attacks. Brain 118:1319-1327

17. Silberstein SD (2004) Migraine pathophysiology and its clinical implications. Cephalalgia 24[Suppl 4]:2-7

18. Bolay H, Reuter U, Dunn AK et al (2002) Intrinsic brain activity triggers trigeminal meningeal afferents in a migraine model. Nat Med 8:136-142

19. Goadsby PJ (2001) Trigeminal autonomic cephalalgias (TACs). Acta Neurol Belg 101:10-19

20. Schoenen J, Ambrosini A, Sandor PS, Maertens de Noordhout AM (2003) Evoked potentials and transcranial magnetic stimulation in migraine: published data and viewpoint on their pathophysiologic significance. Clin Neurophysiol 114:955-972

21. Silberstein SD (2004) Migraine. Lancet 363:381-391

22. Schoenen J (2006) Neurophysiological features of the migraine brain. Neurol Sci Suppl 2:S77-S81

23. Sanchez-del-Rio M, Reuter U, Moskowitz MA (2006) New insights into migraine pathophysiology. Curr Opin Neurol 19:294-298.

24. Dalkara T, Zerues NT, Moskowitz MA (2006) From spreading depression to the trigeminovascular system. Neurol Sci Suppl 2:S86-S90

25. Janig W (2003) The relationship between pain and autonomic phenomena in headache and other pain conditions. Cephalalgia 23[Suppl 1]:43-48
26. Olesen J (2006) Are headache disorders caused by neurobiological mechanisms. Editorial review. Curr Opin Neurol 19:277-280

27. Strong AJ, Fabricius M, Boutelle MG et al (2002) Spreading and synchronous depressions of cortical activity in acutely injured human brain. Stroke 33:2738-2743

28. Hadji Khani N, Sanchez-del-Rio M, Wu O et al (2001) Mechanisms of migraine aura revealed by functional MRI in human visual cortex. Proc Natl Acad Sci U S A 98:4687-4692

29. Reuter U, Bolay H, Jansen-Olesen I et al (2001) Delayed inflammation in rat meninges: implications for migraine pathophysiology. Brain 124:2490-2502

30. Bolay H, Reuter U, Dunn AK et al (2002) Intrinsic brain activity triggers trigeminal meningeal afferent in a migraine model. Nat Med 8:136-142

31. Gursoy-Ozdemir Y, Qiu J, Matsuoka N et al (2004) Cortical spreading depression activates and upregulates MMP-9. J Clin Invest 113:1447-1455

32. Spierings EL (1995) Symptomatology and pathogenesis of migraine. J Pediatr Gastroenterol Nutr 21[Suppl 1]:S37-S41

33. Boccuni M, Alessandri M, Fusco BM, Cangi F (1989) The pressor hyperresponsiveness to phenylephrine unmasks sympathetic hypofunction in migraine. Cephalalgia 9:239-245

34. Haeter K, Ayata C, Moskowitz MA (2005) Cortical spreading depression: a model for understanding migraine biology and future drug targets. Headache Curr 2:97-103
35. Wassermann EM (1998) Risk and safety of repetitive transcranial magnetic stimulation: report and suggested guidelines from the International Workshop on Safety of Repetitive Transcranial Magnetic Stimulation, June 5-7, 1996. Electroencephalogr Clin Neurophysiol 108:1-16

36. Anand S, Hotson J (2002) Transcranial magnetic stimulation: neurophysiological applications and safety. Brain Cogn 50:366-386

37. Headache Classification Committee of the International Headache Society (2004) The International Classification of Headache Disorders, 2nd ed. Cephalalgia 14[Suppl 1]:8-152

38. Clarke BM, Upton ARM, Castellanos CM, Schmuck ML (2005) Measuring and assessing suffering in arthritic patients. In: Meier A, O'Connor T, Vankatwyk PL (eds) Spirituality and health: multidisciplinary explorations. Wilfrid Laureir University Press, Waterloo, Ontario, pp 227-240

39. Malik M, Camm AJ (eds) (1995) Heart rate variability. Futura Publishing Co., New York

40. Appel S, Kuritzky A, Zahavi I et al (1992) Evidence for instability of the autonomic nervous-system in patients with migraine headache. Headache 32:10-17

41. Brandes JL (2003) Treatment approaches to maximizing therapeutic response in migraine. Neurology 61[Suppl 4]:S21-S26

42. Waeber C, Moskowitz MA (2003) Therapeutic implications of central and peripheral neurologic mechanisms in migraine. Neurology 61[Suppl 4]:S9-S20 